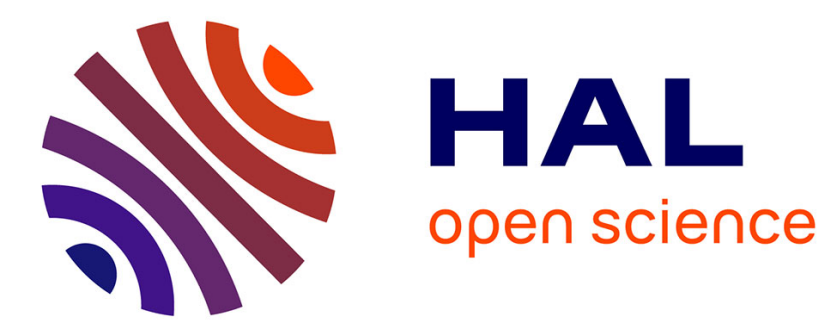

\title{
Étude de la sensibilité des paramètres d'un modèle "rural" sur des bassins versants urbanisés
}

\author{
Mohamed Saadi, Ludovic Oudin, Pierre Ribstein
}

\section{To cite this version:}

Mohamed Saadi, Ludovic Oudin, Pierre Ribstein. Étude de la sensibilité des paramètres d'un modèle "rural" sur des bassins versants urbanisés. La Houille Blanche - Revue internationale de l'eau, 2019, 2, pp.35-43. 10.1051/lhb/2019013 . hal-02301466

\section{HAL Id: hal-02301466 https://hal.sorbonne-universite.fr/hal-02301466}

Submitted on 30 Sep 2019

HAL is a multi-disciplinary open access archive for the deposit and dissemination of scientific research documents, whether they are published or not. The documents may come from teaching and research institutions in France or abroad, or from public or private research centers.
L'archive ouverte pluridisciplinaire HAL, est destinée au dépôt et à la diffusion de documents scientifiques de niveau recherche, publiés ou non, émanant des établissements d'enseignement et de recherche français ou étrangers, des laboratoires publics ou privés. 


\title{
Étude de la sensibilité des paramètres d'un modèle "rural" sur des bassins versants urbanisés
}

\author{
Mohamed Saadi*, Ludovic Oudin, Pierre Ribstein \\ Sorbonne Université, CNRS, EPHE, UMR METIS, F-75000, Paris, France. \\ *Auteur correspondant : mohamed.saadi@upmc.fr
}

\section{Résumé}

Plusieurs modèles peuvent être utilisés pour représenter les processus hydrologiques en milieu urbain. Ces modèles sont développés suivant différentes approches en fonction de l'objectif de modélisation, de l'échelle spatio-temporelle et de la disponibilité des données hydroclimatiques et spatiales. Dans cet article, nous proposons de développer un outil parcimonieux et généralisable à partir d'une structure de modèle non-dédié au contexte urbanisé. Dans un premier temps, nous analysons la structure de départ et la sensibilité des paramètres qu'elle contient à la présence de surfaces urbaines dans le bassin versant. Pour ce faire, un échantillon de 365 bassins versants français et américains a été construit. Le modèle est calé sur deux périodes pour chaque bassin versant, donnant ainsi 730 couples bassin-période pour lesquels la fraction urbaine dans le bassin a été déterminée. L’analyse de la sensibilité des paramètres du modèle hydrologique par rapport à cette fraction ainsi que d'autres indicateurs hydrologiques a été effectuée pour une sélection de 416 couples bassin-période, homogènes en surface. Les résultats montrent que les paramètres du modèle et les indicateurs hydrologiques présentent des différences significatives entre un échantillon de bassins versants fortement urbanisés et un échantillon de bassins versants faiblement urbanisés, traduisant des écoulements plus rapides et plus importants sur les bassins fortement urbanisés.

Mots Clefs : Modélisation hydrologique; Urbanisation; GR4H; Sensibilité.

\section{Sensitivity analysis of a "rural" model parameters on urbanized catchments}

\begin{abstract}
used in order to represent the hydrological processes in an urbanized catchment. Developing those models has been with respect to modeling objectives, spatiotemporal scale and hydroclimatic and spatial data availability. These models can be explicit, hence complex and data demanding, or rather implicit, but lacking direct linking between the model parameters and the urbanization features. Leaning towards simplicity, we aim at developing an efficient modeling tool that permits implicitly to take into account the spatial configuration (urban landscape $v s$. natural surfaces) and temporal evolution at the scale of an urbanized hydrological catchment. Development of such tool can be achieved by complexifying step-by-step a starting model structure that is not dedicated to urbanized contexts. Before conducting structure modifications, the first step to take is to determine
\end{abstract}


which model functions can be modified. In this paper, we analyze the initial structure and the sensitivity of the parameters that it contains to the presence of urban surfaces within the catchment. For this end, a large sample of 365 French and American catchments was prepared. These catchments are of various sizes going from 1 to $5000 \mathrm{~km}^{2}$ and represent a large panel of hydroclimatic conditions. The model was calibrated on two periods for each catchment, leading to 730 catchment-period couples for which the fraction of the urban cover was determined. The sensitivity analysis of the model parameters and other hydrological indicators with respect to this fraction was conducted for a selection of area-homogeneous 416 catchment-period couples. The results of this empirical study have shown (1) good model performances at the hourly time step regardless of the urban condition of the catchment and (2) that the model parameters and the hydrological indicators are significantly different with regards to intensively or slightly urbanized catchments, reflecting faster and greater streamflows for intensively urbanized situations.

Keywords : Hydrological modeling; Urbanization; GR4H; Sensitivity.

\section{Introduction}

Plusieurs approches de modélisation hydrologique existent en hydrologie urbaine pour simuler les écoulements des bassins urbanisés. Le choix d'une approche dépend au premier ordre de l'objectif de modélisation (étude d'impact, dimensionnement, aménagement, etc.), de l'échelle choisie au niveau spatial (quartier, ville ou bassin versant naturel) et temporel (infra-journalière, journalière, mensuelle ou annuelle). Le choix de l'approche est aussi contraint par la disponibilité et la qualité des données hydroclimatiques (notamment les précipitations et les débits) et de caractéristiques du milieu (e.g. l'occupation du sol, les données cadastrales, le maillage du réseau d'assainissement). Si lorsque le bassin versant est urbain, il semble nécessaire de tenir compte de façon fine de ses caractéristiques urbaines (réseau d'assainissement, schéma d'urbanisme, etc.), la question reste très ouverte pour les bassins versants urbanisés pour lesquels des zones urbaines jouxtent des territoires moins anthropisés et souvent plus perméables. Dans ce contexte, nous proposons de développer une modélisation suivant une approche "Top-down », en modifiant la structure d'un modèle hydrologique simple pour tenir compte des spécificités des processus hydrologiques en contexte urbanisé. Ce type de développement nécessite l'utilisation d'un large échantillon de bassins versants, hétérogène en matière de comportement hydrologique ainsi qu'au niveau de l'urbanisation, afin de garantir une certaine robustesse et généralité de la structure obtenue.

Les travaux de modification de structure de modèles pour prendre en compte les caractéristiques de l'urbanisation sont assez courants. Furusho et al. (2013) ont adapté le modèle ISBA-TOPMODEL pour prendre en compte les aspects hydrologiques d'un bassin versant péri-urbain, où l'on rencontre une mosaïque de surfaces urbaines très imperméables et de surfaces naturelles. Les caractéristiques de l'urbanisation inclues dans le modèle étaient le drainage des eaux souterraines par le réseau d'assainissement et la présence d'un réseau d'écoulement préférentiel mis en place par les réseaux urbains qui drainent les surfaces imperméables connectées. Kjeldsen et al. (2013) ont amélioré les performances du modèle conceptuel et global ReFH sur des bassins versants urbanisés. L'estimation des paramètres a été modifiée pour prendre en compte le degré d'urbanisation du bassin étudié. Singh et al. (2009) ont développé un modèle SIMHYD introduisant, dans une structure de modèle hydrologique non-dédié aux bassins urbanisés, plusieurs réservoirs et canalisations permettant de prendre en compte certains processus anthropiques, comme l'irrigation, l'alimentation en eau potable, l'assainissement etc.

Ces tentatives de modification présentent le point faible d'être appliquées sur un nombre limité de bassins versants, ce qui pose question sur l'application de ces modèles en dehors des bassins versants utilisés pour les développer. Dans cette étude, nous proposons d'étudier un large échantillon de bassins versants pour effectuer des tests dans des conditions très variées (Gupta et al., 2014). 
Avant d'entamer le travail de modification d'un modèle, une analyse de la structure de départ et de la sensibilité de ses paramètres vis-à-vis des aspects de l'urbanisation semble une étape primordiale pour identifier les éléments susceptibles d'être modifiés. Dans cette optique, nous avons fait le choix de partir d'un modèle " rural » et d'analyser sa structure sur un échantillon présentant un large gradient d'urbanisation. L'objectif de cet article est donc d'analyser les différences qui peuvent exister dans les paramètres du modèle lorsqu'il est appliqué sur des bassins versants faiblement/fortement urbanisés, de superficies s'étalant entre 1 et $10^{4} \mathrm{~km}^{2}$.

\section{Outils et méthodes}

\subsection{Modèle hydrologique}

Le modèle utilisé est GR4H-I, Génie Rural à 4 paramètres au pas de temps Horaire comprenant un réservoir d'Interception (Ficchi, 2017; Le Moine, 2008; Mathevet, 2005). Le fonctionnement du modèle est schématisé dans la figure 1.

Le niveau actualisé du réservoir d'interception $I[\mathrm{~mm}]$ est soumis aux forçages atmosphériques matérialisés par les précipitations $P$ et l'évapotranspiration potentielle $E$. Il diminue en lui amputant une quantité deau $E_{i}$ pour satisfaire $E$. Il augmente en l'alimentant par les précipitations $P$. Dans les deux cas, $I$ ne peut être ni inférieur à 0 ni supérieur à la capacité maximale $I_{\max }[\mathrm{mm}]$.

Lorsque $E$ n'est pas complètement satisfaite par le contenu du réservoir d'interception, une quantité d'eau $E_{s}$ est retranchée du réservoir de production. Elle est fonction de $E-E_{i}, S[\mathrm{~mm}]$ et $X_{1}[\mathrm{~mm}]$, où $S$ est le niveau actualisé du réservoir de production qui ne peut pas dépasser sa capacité maximale $X_{1}$. Par ailleurs, l'excédent du réservoir d'interception $P_{\text {th }}$ alimente en partie le réservoir de production par une quantité $P_{s}$, qui est fonction de $P_{t h}, S$ et $X_{1}$.

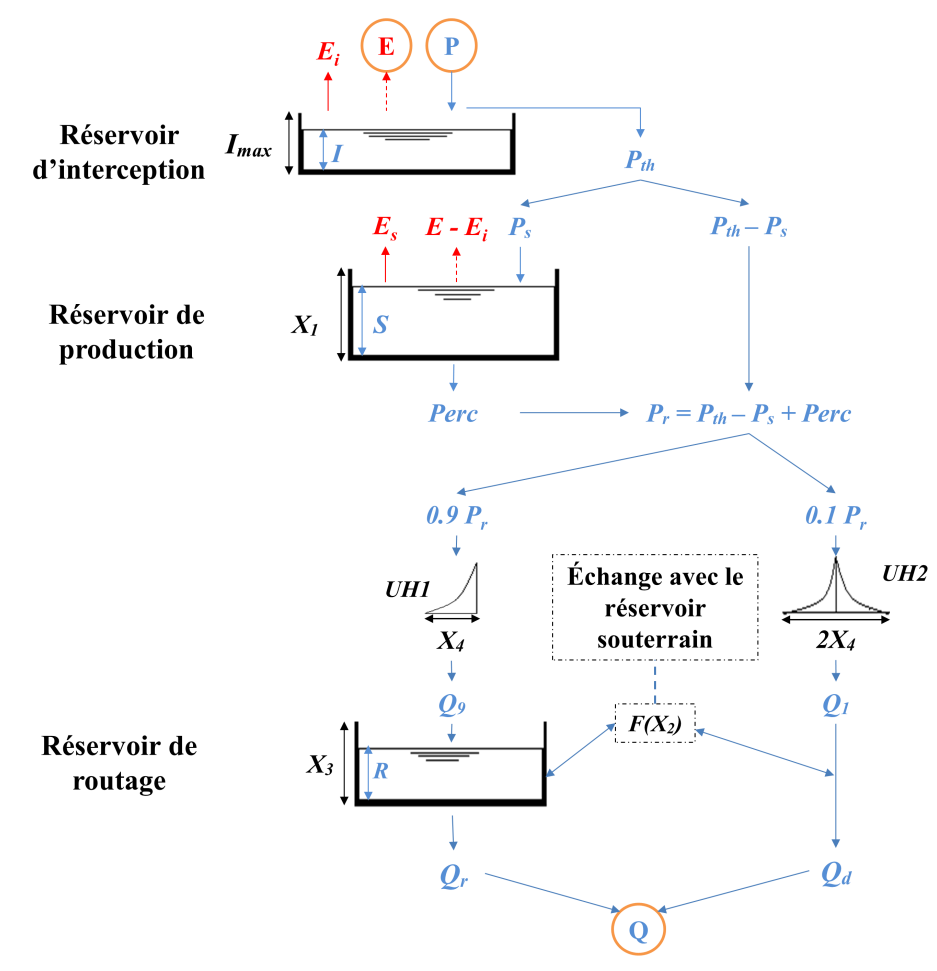

Figure 1 - Structure du modèle GR4H-I, d'après Ficchi (2017). 
Une partie du contenu du réservoir de production contribue à l'entrée de la fonction de routage. Il s'agit de la lame d'eau percolée Perc. La sortie de la fonction de production $P_{r}$ est ensuite divisée en deux pour être transférée via un hydrogramme unitaire UH2 de temps de base $2 X_{4}$, et via une branche \{hydrogramme unitaire $U H 1+$ un réservoir non linéaire\}. La capacité du réservoir de routage non linéaire $X_{3}[\mathrm{~mm}]$ et le temps de base $X_{4}[h]$ de l'hydrogramme $U H 1$ constituent deux paramètres du modèle.

Avant d'arriver au débit de sortie, une fonction d'échange nappe-rivière $F\left(X_{2}\right)$ est déterminée et dépend du paramètre $X_{2}[\mathrm{~mm} / \mathrm{h}]$ qui est positif en cas d'apport d'eau vers la rivière (augmentation du niveau $R[\mathrm{~mm}]$ du réservoir et du débit $Q_{1}$ à la sortie de l'hydrogramme $U H 2$ ) et négatif en cas d'export vers le réservoir souterrain.

On recense au final 4 paramètres dans le modèle qui doivent être optimisés : deux jouant un rôle important pour le bilan hydrique $\left(X_{1}[\mathrm{~mm}]\right.$ du réservoir de production et $X_{2}[\mathrm{~mm} / \mathrm{h}]$ de la fonction d'échange) et deux autres dont le but est de reproduire la dynamique de la réponse du bassin versant $\left(X_{3}[\mathrm{~mm}]\right.$ du réservoir de routage et $X_{4}[h]$ qui contrôle le temps de base des hydrogrammes unitaires). Dans cette version du modèle, le paramètre $I_{\max }$ du réservoir d'interception est fixé. Il est choisi dans une gamme de 18 valeurs allant de $0.1 \mathrm{~mm}$ à $15 \mathrm{~mm}$ de façon à avoir le même cumul de précipitations non-interceptées au pas temps horaire qu'au pas de temps journalier (Ficchi, 2017).

Ce modèle a été conçu et amélioré à travers plusieurs études antérieures utilisant de larges échantillons de bassins versants en majorité ruraux (e.g. Ficchi, 2017; Le Moine, 2008; Mathevet, 2005). Sa structure est parcimonieuse et ne demande comme forçages que les précipitations et l'évapotranspiration potentielle au pas de temps horaire. En contexte urbanisé, on s'attend à ce que le paramètre $X_{1}$ et les paramètres de routage $X_{3}$ et $X_{4}$ soient sensiblement affectés à cause d'une part de la diminution de capacité de rétention du bassin urbanisé (effet d'imperméabilisation des surfaces) et d'autre part de l'augmentation de la rapidité des cours d'eau urbains (Diem et al., 2018) ce qui affecte le temps de réponse du bassin.

\subsection{Données}

Un échantillon de 365 bassins versants urbanisés situés aux États-Unis et en France a été constitué.

L'échantillon américain contient 271 bassins versants (figure 2) sélectionnés à partir de la base de données GAGE-II de l'USGS (United States Geological Survey) (Falcone, 2011) en répondant à des critères de limitation de la présence de la neige (la part des précipitations considérée comme neige doit être inférieure à 10\%) et de l'impact des ouvrages de régulation ou de rétention (le rapport du volume total stocké dans ces ouvrages sur le volume de l'écoulement annuel moyen du bassin doit être inférieur à 5\%), et en ayant au moins 10\% de la surface du bassin versant urbanisée en 2006 . Les données couvrent au minimum 8 ans entre 2002 et 2013 et contiennent des précipitations radar corrigées (NEXRAD - Stage IV, Horvat et al. (2011)), à une résolution de $4 k m$ (Hardegree et al., 2008), et des données d'évapotranspiration potentielle calculées à l'aide des températures moyennes journalières extraites de la base de données de Livneh et al. (2015) et moyennant la formule d'Oudin et al. (2005). Les données débitmétriques sont préparées à partir des mesures instantanées du réseau de jaugeage de l'USGS.

Léchantillon des bassins versants français contient 94 bassins versants (figure 3) dont les données couvrent au minimum 8 ans entre 1997 et 2007. Les critères de sélection préliminaire étaient de disposer des données de débit au pas de temps horaire et d'avoir au minimum $10 \%$ de la surface du bassin versant urbanisée en 2012, en plus de la limitation de la présence de la neige et de l'impact des ouvrages de régulation. Les données de précipitations sont extraites du produit COMEPHORE (résolution de $1 \mathrm{~km}$ ) de Météo-France. Les données de débit sont extraites à partir de la Banque HYDRO. Les températures moyennes journalières permettant le calcul de l'évapotranspiration potentielle par la formule d'Oudin et al. (2005) sont extraites du produit SAFRAN (sur des mailles de $8 * 8 \mathrm{~km}^{2}$ ) de Météo-France. 


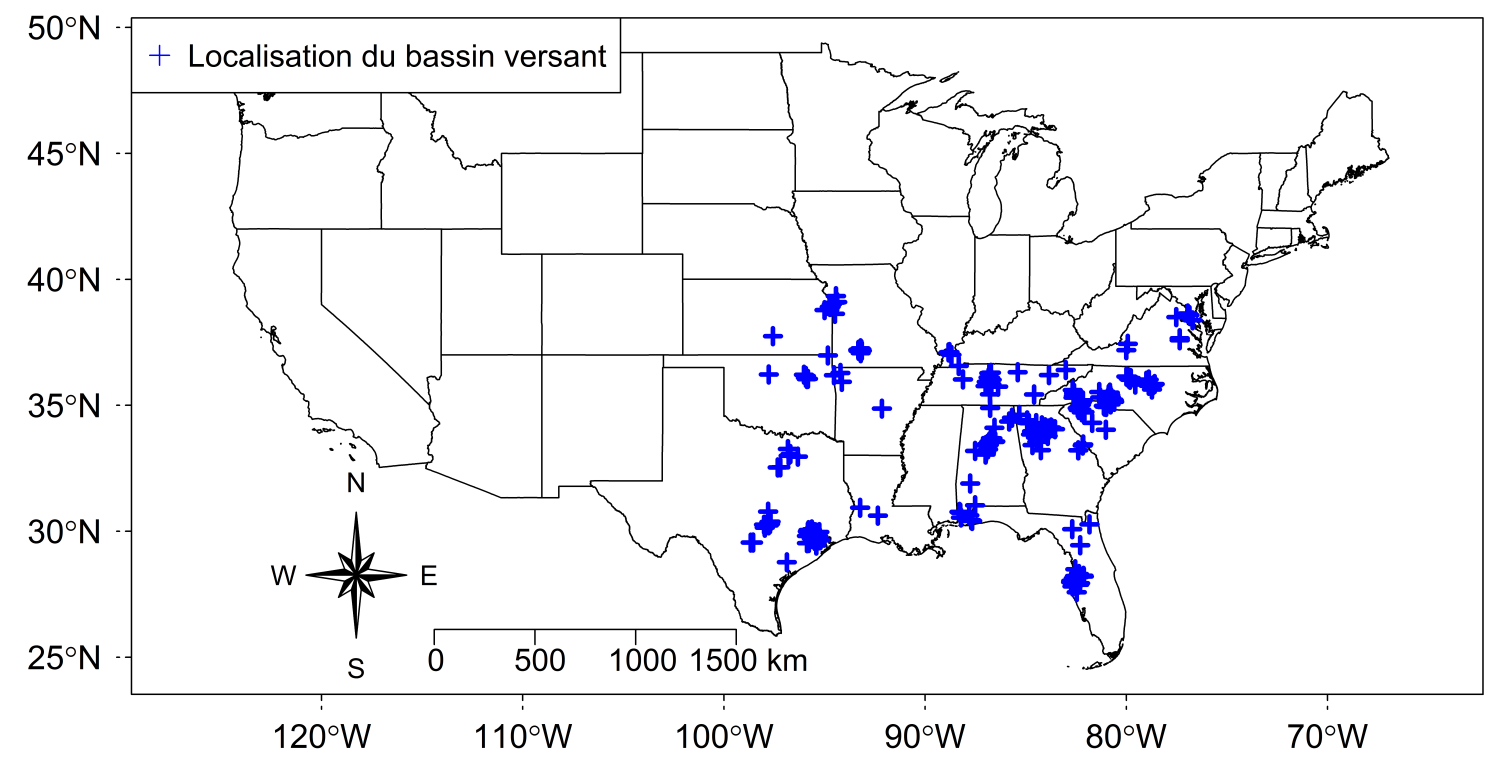

Figure 2 - Localisation des 271 bassins versants américains. Les croix bleues représentent les centroïdes des bassins versants.

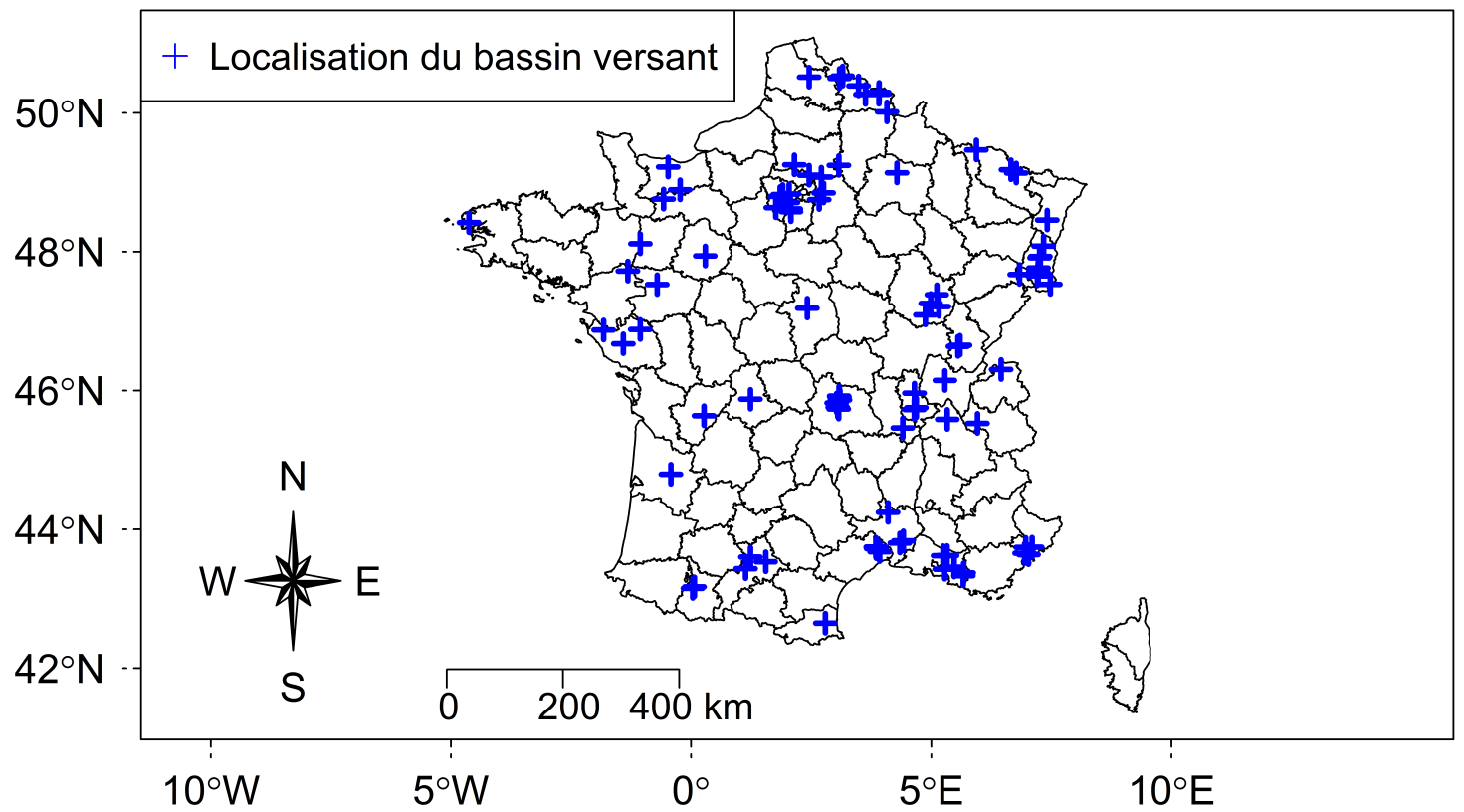

Figure 3 - Localisation des 94 bassins versants français. Les croix bleues représentent les centroïdes des bassins versants.

Le pourcentage urbanisé du bassin versant, $C P D$ «Catchment Percent Developed ", est utilisé pour représenter la part du bassin versant occupée par des classes d'urbanisation (rapport du nombre des pixels urbanisés sur le nombre total des pixels). La base de données NLCD (National Land Data Cover, disponible sur https ://www.mrlc.gov, e.g. Homer et al. (2004)), qui est disponible sur les États-Unis pour 2001, 2006 et 2011, a été utilisée pour calculer le $C P D$ pour les bassins versants américains, en considérant la somme des classes suivantes : urbanisé espace ouvert (21: developed open space), urbanisé à faible intensité (22 : developed low intensity), urbanisé à moyenne intensité (23: developed medium intensity) et urbanisé à forte intensité ( 24 : developed high inten- 
sity). Quant aux bassins versants français, la base de données CLC (Corine Land Cover, disponible sur https :/ / land.copernicus.eu / pan-european/corine-land-cover) disponible sur l'Europe en 1990, 2000, 2006 et 2012 a été utilisée. Dans ce cas-là, les classes considérées sont : tissu urbain continu (111 : continuous urban fabric), tissu urbain discontinu (112 : discontinuous urban fabric), unités industrielles ou commerciales (121 : industrial or commercial units), routes, chemins de fer et terrains associés (122 : road and rail networks and associated land), aires portuaires (123: port areas) et aéroports (124 : airports). Le CPD varie de $0 \%$ pour un bassin sans présence d'urbanisation jusqu'à $100 \%$ pour un bassin complètement urbanisé. Même si cette mesure simple d'urbanisation ne permet pas de donner une indication sur la typologie d'urbanisation du bassin versant ou sur l'éventuelle connexion des surfaces urbaines au réseau de drainage naturel ou artificiel, elle représente la variable disponible pour l'ensemble des bassins et elle est relativement homogène entre les bassins situés en France et aux États-Unis.

\subsection{Méthodologie}

Le modèle est calé sur deux périodes de 4 ans : une au début de la chronique et une autre à la fin. Une année de plus est rajoutée pour la mise en route du modèle. On calcule le $C P D$ correspondant à chaque période. Pour un nombre $\mathrm{N}$ de bassins versants, on construit donc un nombre $2 N$ de couples $(C P D, X)$ avec $X=\left\{X_{1}, X_{2}, X_{3}, X_{4}\right\}$. Pour chacune des périodes, on calcule trois autres caractéristiques : le rapport $\frac{Q}{P}$ qui renseigne sur le rendement des bassins versants, l'indice d'écoulement de base BFI déterminé par la méthode de Gustard et al. (1992) qui renseigne sur la partition entre écoulements lent et rapide, et le temps de réponse du bassin défini comme le décalage $D T$ (en heures) à effectuer sur la chronique de débit (ou de pluie) pour maximiser le coefficient de corrélation pluie-débit. Le but d'utiliser des indicateurs hydrologiques est de permettre d'une part une synthèse du comportement des bassins versants, et d'autre part de voir si un changement de comportement qui apparaît dans les indicateurs se traduit dans les paramètres du modèle $\left(\frac{Q}{P}\right.$ vs. $X_{1}, B F I$ vs. $\left\{X_{2}, X_{3}\right\}$ et $D T$ vs. $\left\{X_{3}, X_{4}\right\}$ ).

Le calage est effectué en utilisant un algorithme constitué de deux étapes, d'abord une recherche globale sur une grille grossière de paramètres identifie le meilleur point de départ, puis à partir de ce point un algorithme de gradient présenté par Edijatno et al. (1999) permet une recherche plus locale du jeu de paramètres optimisant le coefficient de Kling-Gupta (Gupta et al., 2009) KGE, exprimé par :

$$
K G E=1-\left((1-r)^{2}+(1-\alpha)^{2}+(1-\beta)^{2}\right)^{0.5}
$$

Où $r$ est la corrélation entre le débit simulé et observé, $\alpha$ le rapport de leurs écarts-types et $\beta$ le rapport de leurs moyennes. Le score $K G E$ prend des valeurs entre $-\infty$ et 1,1 étant la valeur idéale. Ce calage a été effectué en utilisant le package airGR (Coron et al., 2018) dans un environnement de développement en $\mathrm{R}$ ( $\mathrm{R}$ Core Team, 2017).

\section{Résultats}

\subsection{Performances du modèle}

Les valeurs de médianes des scores $K G E$ (figure 4) sont satisfaisantes pour les 730 périodes de calage (188 en France et 542 en US). Sur quelques périodes, les performances sont faibles, surtout pour l'échantillon français, où l'on observe légèrement plus de variabilité de performance du modèle par rapport au cas américain. Les médianes sont $0.85,0.84$ et 0.85 respectivement pour les bassins 
français, américains et pour l'échantillon global. On remarque également que les performances du modèle sont aussi bonnes sur l'échantillon fortement urbanisé (URB pour les couples bassin-période avec valeurs de $C P D$ supérieures à la médiane des $C P D$ qui est égale à $27.1 \%$ ) que sur l'échantillon faiblement urbanisé (NURB pour les couples bassin-période avec valeurs de $C P D$ inférieures à la médiane) avec une différence en médiane de 0.03 seulement. Les performances du modèle ne sont ainsi pas sensibles à la valeur du paramètre $C P D$ (corrélation $(K G E, C P D)=-0.092)$.

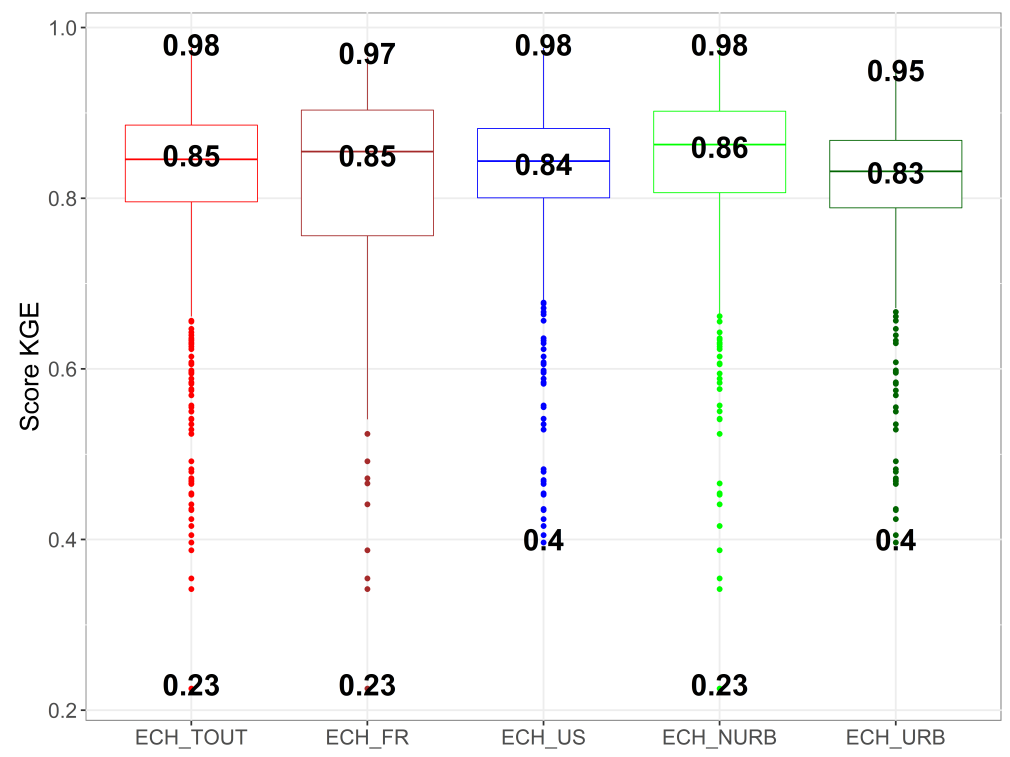

Figure 4 - Scores $K G E$ du calage du modèle effectué sur les différentes périodes pour l'échantillon total (ECH_TOUT), l'échantillon de bassins français $\left(E C H \_F R\right)$, l'échantillon de bassins versants américains ( $\left.E C H \_U S\right)$, l'échantillon faiblement urbanisé ( $E C H \_N U R B$ ) et l'échantillon fortement urbanisé. Dans l'ensemble des figures contenant des boites à moustaches, les chiffres indiquent les valeurs du minimum, de la médiane et du maximum, et les boites sont délimitées par les quantiles $25 \%$ et $75 \%$ de chacun des échantillons.

\subsection{Distribution des valeurs de $C P D$ et différences France/États-Unis}

Le tableau 1 indique les moyennes de surface $\left(S_{\text {moy }}\right.$ en $\left.\mathrm{km}^{2}\right)$ et de quelques caractéristiques hydroclimatiques (précipitation $P_{m o y}$, évapotranspiration potentielle $E T P_{m o y}$ et débit à l'exutoire $Q_{m o y}$ en $\mathrm{mm} / \mathrm{h}$ ) calculées sur les périodes 2002-2013 pour l'échantillon américain et 1997-2007 pour l'échantillon français. Les bassins versants français sont en moyenne plus grands que les bassins versants américains. Des différences en matière de forçage climatique existent entre les deux pays : les précipitations et l'évapotranspiration potentielle moyennes sont légèrement plus importantes pour l'échantillon américain. Cette légère différence se traduit par une différence en termes d'écoulement moyen observé à l'exutoire, qui est plus important pour l'échantillon américain.

\begin{tabular}{cccc}
\hline & Tout & FR & US \\
\hline$S_{\text {moy }}\left[\mathrm{km}^{2}\right]$ & 284.47 & 323.21 & 271.04 \\
$E T P_{\text {moy }}[\mathrm{mm} / \mathrm{h}]$ & 0.123 & 0.09 & 0.135 \\
$P_{\text {moy }}[\mathrm{mm} / \mathrm{h}]$ & 0.126 & 0.096 & 0.137 \\
$Q_{\text {moy }}[\mathrm{mm} / \mathrm{h}]$ & 0.048 & 0.038 & 0.051 \\
\hline
\end{tabular}

Tableau 1 - Caractéristiques moyennes pour tout l'échantillon, en France et aux États-Unis.

On remarque sur la figure 5 que l'on rencontre des valeurs de $C P D$ plus importantes dans l'échantillon américain, avec une médiane de $45.6 \%$, alors que les bassins versants français ont des valeurs 
de $C P D$ plus faibles. Les bassins versants américains ont été sélectionnés de manière à avoir en 2006 un $C P D$ plus grand ou égal à 10\%, alors que cette condition n'est appliquée sur l'échantillon français qu'en 2012. De plus, on remarque que l'échantillon français est plus homogène que l'américain, avec $50 \%$ des valeurs de $C P D$ dans l'intervalle $[10 \% ; 21 \%]$ contre $[18 \% ; 82 \%]$ pour l'échantillon américain.

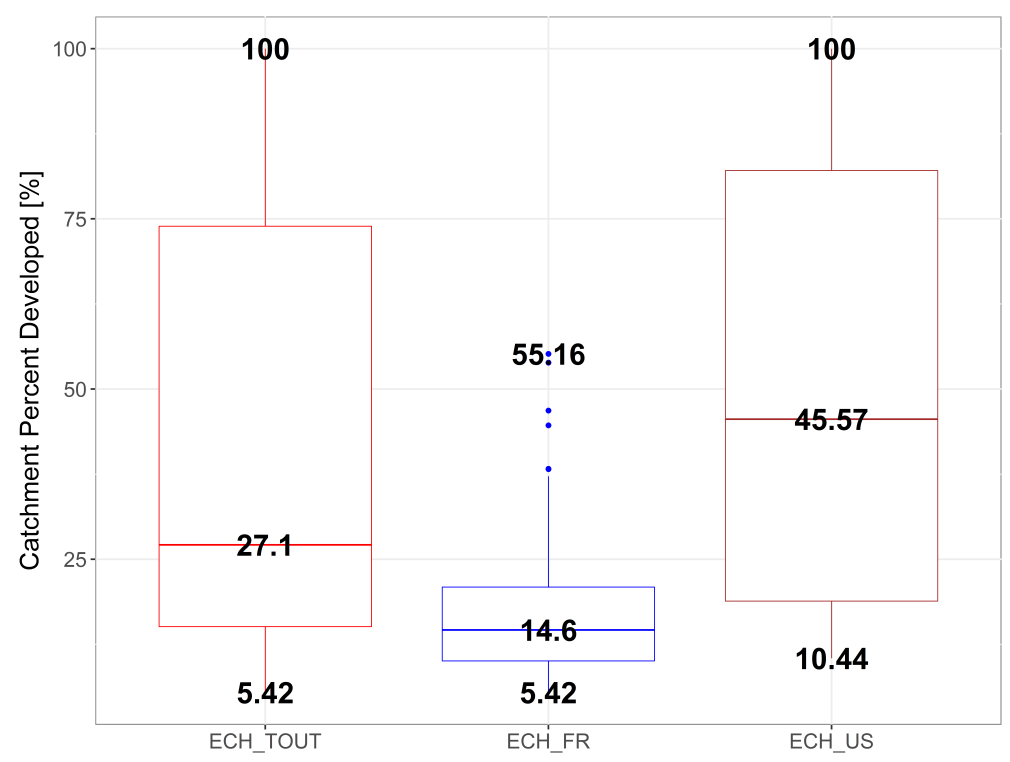

Figure 5 - Distribution des valeurs du $C P D$ pour l'échantillon total, l'échantillon des bassins versants français et l'échantillon des bassins versants américains.

Malgré la simplicité de cette mesure, le $C P D$ peut donner une idée sur la différence d'urbanisation entre les deux échantillons. En revanche, elle est très influencée par la surface. En divisant les périodes en deux sous-échantillons fortement urbanisé (URB)/faiblement urbanisé (NURB) par la médiane des valeurs $C P D(27.1 \%)$, on remarque que, généralement, les faibles valeurs de $C P D$ (i.e. échantillon faiblement urbanisé) sont rencontrées sur des bassins versants plus grands (figure 6(a)). Cette différence de surface entre les deux échantillons pourrait biaiser l'interprétation des résultats, étant donné que la dynamique de la réponse est également influencée par la surface du bassin. Ainsi, nous avons pu constituer un échantillon plus restreint de bassins-périodes (416 au lieu de 730) en sélectionnant des bassins versants de surface homogène entre les deux catégories URB/NURB. Ceci conduit à réduire la diversité des situations mais permet une analyse des résultats non biaisée par la surface. La médiane des valeurs $C P D$ du nouvel échantillon est proche de la médiane de l'échantillon initial (27.1\%). On peut remarquer que les distributions de surfaces entre les deux nouveaux sous-échantillons ne sont pas significativement différentes (figure 6(b)). Il y a au final 112 couples bassins-période situés en France (9 en URB et 103 en NURB) et 304 situés aux États-Unis (199 en URB et 105 en NURB).

\subsection{Différences en termes d'indicateurs hydrologiques}

En parallèle du calage et de l'analyse des paramètres du modèle, des indicateurs hydrologiques sont calculés sur les périodes de calage : le rapport $\frac{Q}{P}$ pour avoir une idée du rendement des bassins versants, l'indice d'écoulement de base BFI et le temps de réponse du bassin $D T$. Sur la figure 7 , on peut visualiser les distributions des valeurs de ces indicateurs pour les deux échantillons de bassins-périodes fortement/faiblement urbanisés, homogènes en termes de surface (comme montré sur la figure 6(b)).

Pour le rapport $\frac{Q}{P}$ (figure $7(a)$ ), on remarque que l'on a plus d'écoulement pour l'échantillon forte- 

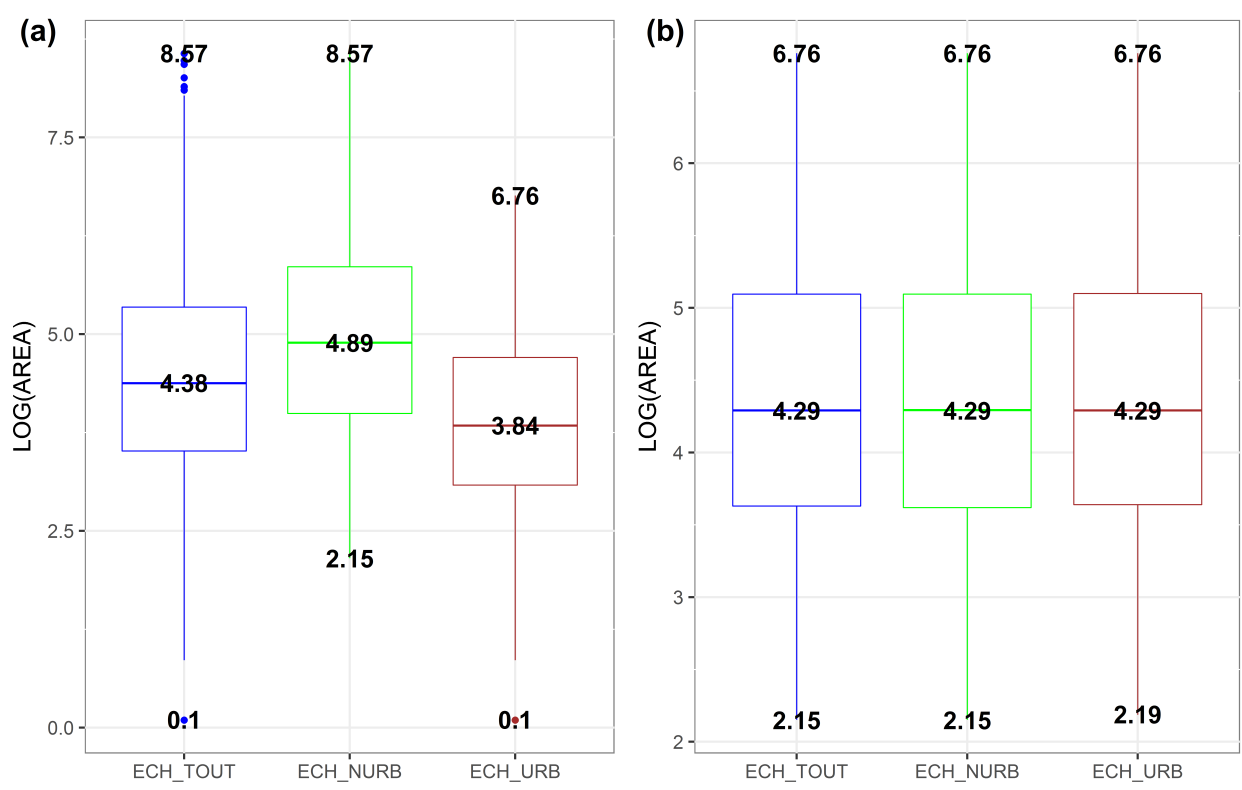

Figure 6 - Distribution des logarithmes des surfaces (en $\mathrm{km}^{2}$ ) des échantillons global, faiblement urbanisé et fortement urbanisé (a) avant homogénéisation et (b) après homogénéisation.
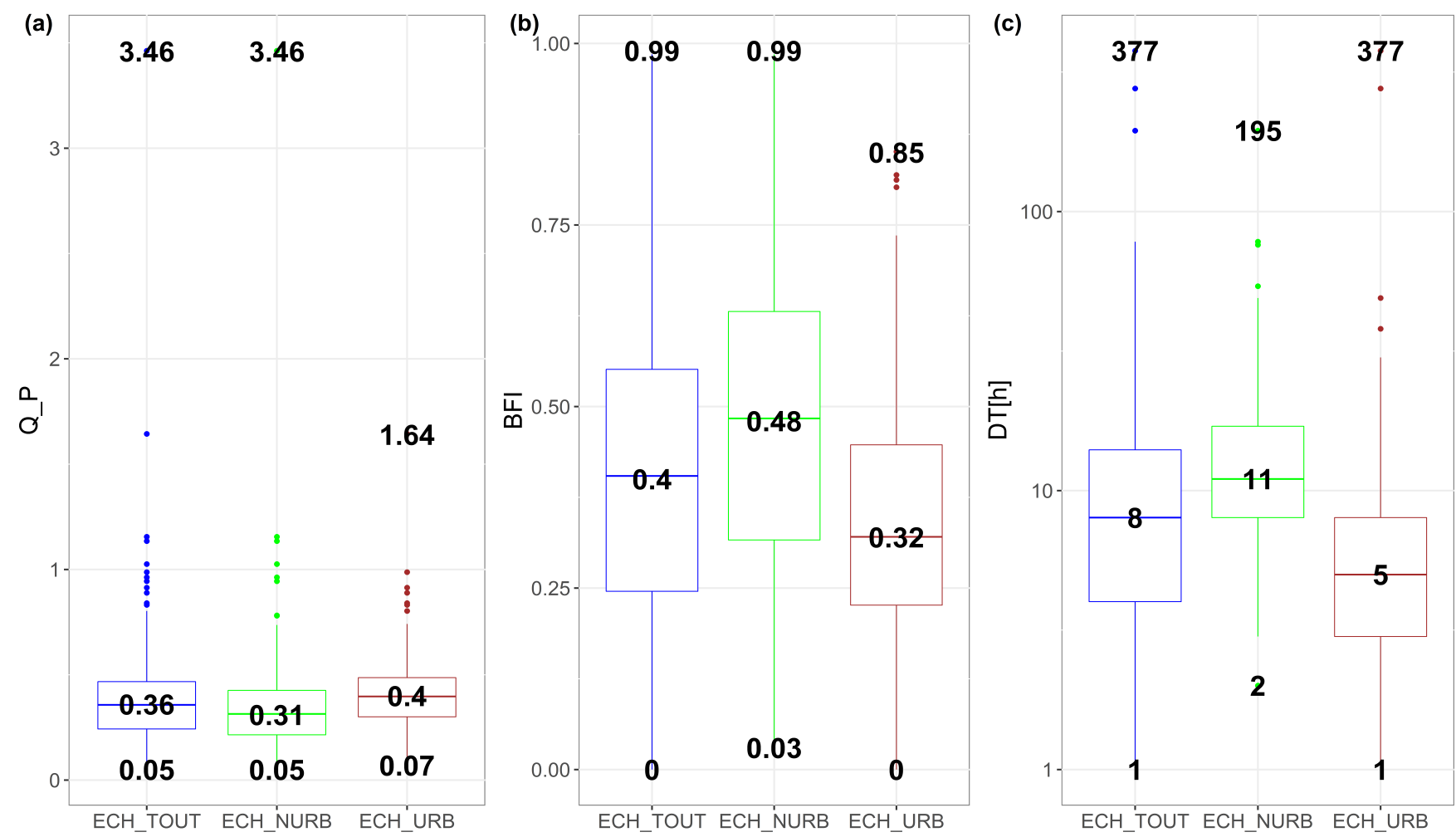

Figure 7 - Distributions des valeurs de (a) $\frac{Q}{P}$, (b) BFI et (c) $D T[h]$ pour les échantillons global, faiblement urbanisé et fortement urbanisé.

ment urbanisé (différence en médiane de 9\%), ce qui est souvent constaté dans la littérature (e.g. Burns et al., 2005; Diem et al., 2018; Hawley and Bledsoe, 2011; Oudin et al., 2018; Rose and Peters, 2001; Salavati et al., 2015). Sur certains couples bassin-période, le rapport $\frac{Q}{P}$ dépasse la valeur maximale de 1 , ce qui pourrait correspondre à des apports importants des réservoirs souterrains (cas de la Touvre avec une valeur de $\frac{Q}{P}=3.46$, station R2335050, voir Le Moine et al. (2008)), 
ou de stations proches des villes (par exemple, la station USGS 08116400 Dry Creek near Rosenberg, dont le bassin versant draine une partie de la ville de Rosenberg, Texas avec une valeur de $C P D=52 \%$ ). Le $B F I$ donne une idée sur la contribution du réservoir souterrain dans l'écoulement observé à l'exutoire : l'échantillon faiblement urbanisé conserve une contribution plus importante que l'échantillon fortement urbanisé (figure 7(b)), ce qui suggère que l'imperméabilisation en surface réduit la recharge des aquifères (e.g. Braud et al., 2013; Diem et al., 2018; Mejía et al., 2015; Rose and Peters, 2001). Le temps de réponse calculé par corrélation pluie-débit est faible pour l'échantillon fortement urbanisé : une médiane de 5 heures contre 11 heures pour l'échantillon faiblement urbanisé (figure $7(\mathrm{c})$ ), ce qui suggère que la dynamique de la réponse est plus rapide en présence d'un fort couvert urbain.

\subsection{Comparaison entre les valeurs des paramètres des deux échantillons}

Sur la figure 8, les distributions des transformées des paramètres du modèle sont montrées pour l'échantillon global, faiblement urbanisé et fortement urbanisé. Le choix de ces transformées a été effectué de manière à normaliser le plus possible les distributions des paramètres. On peut remarquer qu'il existe des différences entre les deux échantillons fortement et faiblement urbanisé. Elles sont plus accentuées pour le paramètre $X_{1}$ (médiane des bassins faiblement urbanisés de $235.8 \mathrm{~mm}$ contre $128.66 \mathrm{~mm}$ pour les bassins fortement urbanisés) et le paramètre $X_{4}$ (médiane des bassins faiblement urbanisés de $13 h$ contre $6 h$ pour les bassins fortement urbanisés). Le paramètre $X_{3}$ se trouve légèrement plus faible sur l'échantillon fortement urbanisé par rapport à l'échantillon faiblement urbanisé.
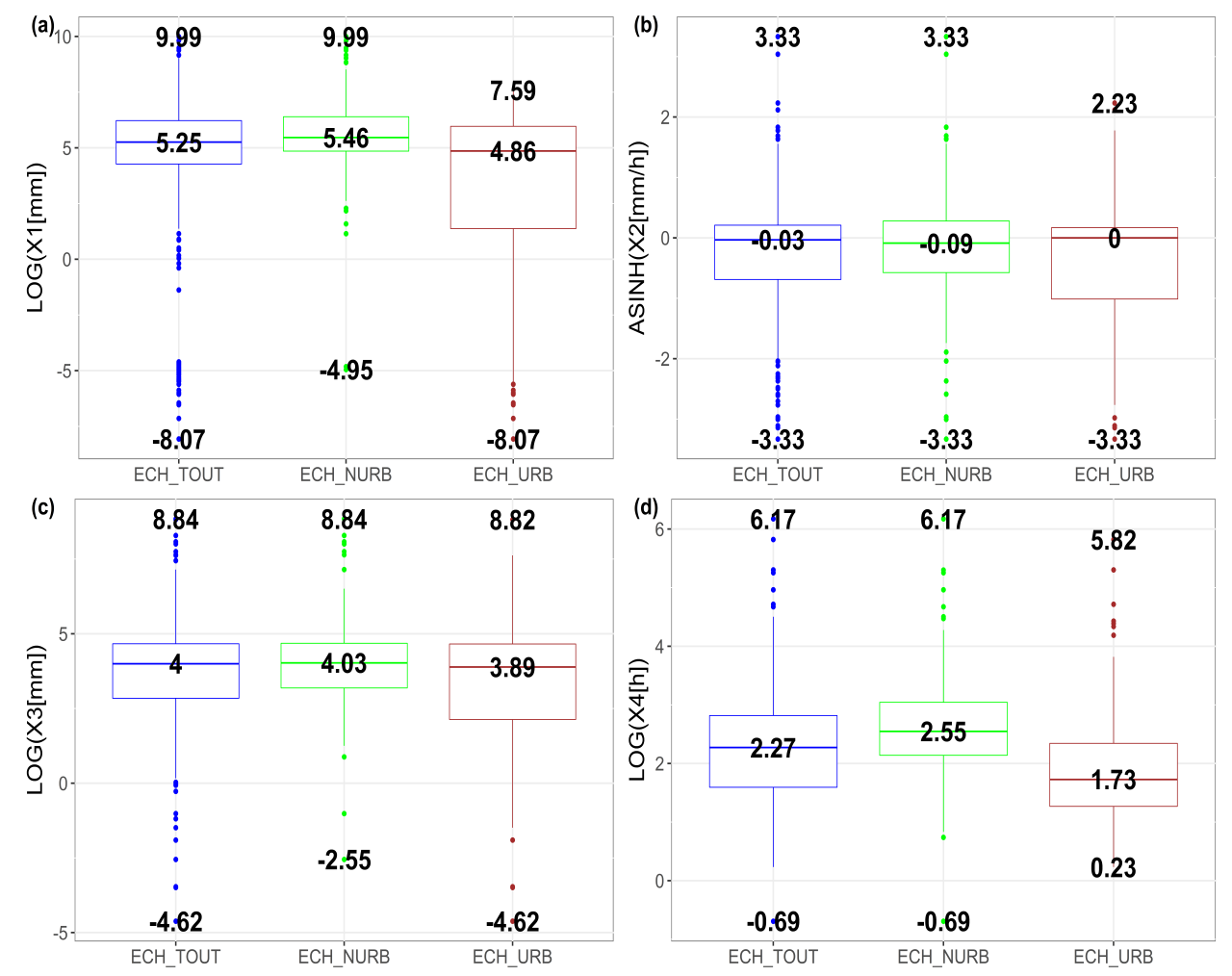

Figure 8 - Distributions des logarithmes des valeurs des paramètres (a) $X_{1}[\mathrm{~mm}]$, (c) $X_{3}[\mathrm{~mm}]$ et (d) $X_{4}[\mathrm{~mm}]$ et de l'argument sinus hyperbolique des valeurs du paramètre $X_{2}[\mathrm{~mm} / \mathrm{h}]$ pour les échantillons global, faiblement urbanisé et fortement urbanisé. 


\subsection{Tests statistiques d'égalité de moyennes}

Le tableau 2 montre les résultats des tests d'égalité de moyennes (Student) des paramètres et indicateurs pour les deux échantillons de bassins versants fortement ou faiblement urbanisés.

\begin{tabular}{ccccc}
\hline & p-value & $\begin{array}{c}\text { Moyenne } \\
\text { NURB }\end{array}$ & $\begin{array}{c}\text { Moyenne } \\
\text { URB }\end{array}$ & $\begin{array}{c}\text { Moyennes } \\
\text { égales? (au seuil } \\
\text { de 0.05) }\end{array}$ \\
\hline LOG $\left(\right.$ AREA $\left.\left[\mathrm{km}^{2}\right]\right)$ & 0.971 & 4.372 & 4.368 & OUI \\
\hline LOG $\left(X_{1}[\mathrm{~mm}]\right)$ & $3 * 10^{-13}$ & 5.53 & 2.973 & NON \\
\hline ARCSINH $\left(X_{2}[\mathrm{~mm} / \mathrm{h}]\right)$ & 0.011 & -0.149 & -0.391 & NON \\
\hline LOG $\left(X_{3}[\mathrm{~mm}]\right)$ & $2 * 10^{-5}$ & 4.036 & 3.316 & NON \\
\hline LOG $\left(X_{4}[\mathrm{~h}]\right)$ & $3 * 10^{-15}$ & 2.63 & 1.917 & NON \\
\hline$B F I$ & $3 * 10^{-11}$ & 0.479 & 0.345 & NON \\
\hline$\frac{Q}{P}$ & 0.037 & 0.357 & 0.405 & NON \\
\hline LOG $(D T[\mathrm{~h}])$ & $2 * 10^{-19}$ & 2.412 & 1.619 & NON
\end{tabular}

Tableau 2 - Récapitulatif des tests d'égalité de moyennes des sous-échantillons faiblement urbanisé et fortement urbanisé. Pour certaines variables, les tests sont effectués sur des transformées des valeurs afin de normaliser le plus possible les distributions.

Compte tenu des tests sur l'homogénéité des deux sous-échantillons (tests d'égalité de variances, non montré ici), les tests statistiques montrent que les échantillons marquent des différences en termes d'indicateurs hydrologiques mais aussi en termes de paramètres, et ce pour un maximum de risque d'égalité de moyennes de $5 \%$.

\section{Discussion et conclusions}

\subsection{Est-ce que les aspects de l'urbanisation sont bien captés par les paramètres?}

Le modèle utilisé dans cette étude n'a pas été développé pour les bassins versants urbanisés. En essayant de l'appliquer sur des bassins urbanisés, on teste si la différence fortement/faiblement urbanisé peut être détectée par les paramètres du modèle. Le modèle a montré de bonnes performances sur l'ensemble des périodes du calage (figure 4), et ce quel que soit le niveau d'urbanisation du bassin (corrélation $(K G E, C P D)=-0.092)$. Le paramètre de production $X_{1}$ (capacité maximale du réservoir de production) et de routage $X_{4}$ (temps de base des hydrogrammes unitaires) étaient ceux pour lesquels on a pu remarquer les plus grandes différences entre bassins fortement urbanisés et faiblement urbanisés.

Les différences en matière de dynamique sont confirmées par le temps de réaction $D T$ calculé sur les périodes étudiées, où l'on constate un écart évalué à 6 heures en moyenne. Le résultat est assez intuitif; même si les études sur l'impact de l'urbanisation ne sont pas toujours convergentes sur cette question (Oudin et al., 2018), la rapidité des écoulements induite par la mise en place de surfaces urbaines contribue généralement à la diminution de temps de réponse hydrologique (Diem et al., 2018). Cette différence de 6 à 7 heures en moyenne est sensiblement la même que la différence des moyennes du paramètre $X_{4}$. Par ailleurs, la différence rencontrée dans les valeurs de la capacité du réservoir du routage $X_{3}$ peut être expliquée les différences en termes d'indice de $B F I$, où l'on observe des valeurs plus faibles pour les bassins les plus urbanisés, ce qui tend à confirmer que la recharge de l'aquifêre est moins soutenue sur ces bassins. La différence entre les rapports $\left(\frac{Q}{P}\right)_{U R B}$ et $\left(\frac{Q}{P}\right)_{N U R B}$ suggère que le rendement est aussi affecté par l'urbanisation. Le fait que le paramètre 
$X_{1}$ soit plus faible sur les bassins urbanisés suggère que le modèle fait circuler moins d'eau dans le réservoir de production, ce qui a pour effet de modifier le rendement en diminuant les pertes par évaporation mais aussi la dynamique en favorisant les écoulements rapides.

\subsection{Limites de la méthodologie}

Cette étude présente plusieurs limitations. En premier lieu, l'analyse des paramètres a été effectuée sur l'hypothèse que ces paramètres traduisent un aspect de la réalité physique, ce qui est discutable compte tenu de l'empirisme du modèle utilisé. La cohérence entre rôle des paramètres et caractéristiques hydrodynamiques de l'écoulement suggère cependant qu'il est possible d'attribuer un rôle spécifique à chacun des paramètres, ce qui ouvre la voie à des recherches de détermination des valeurs de paramètres en fonction de l'urbanisation du bassin versant.

La simplicité de la mesure d'urbanisation utilisée $(C P D)$ a limité l'interprétation et l'analyse des résultats. Cette mesure ne permet pas de donner une idée sur l'agencement spatial des surfaces urbaines par rapport au réseau hydrographique (imperméabilité effective), ou sur la présence de réseaux d'écoulement artificiels influençant les bilans hydrologiques. En outre, le seuil pris égal à la médiane des valeurs $(C P D$ à $27.1 \%)$ pour distinguer un échantillon fortement urbanisé d'un échantillon faiblement urbanisé est arbitraire, et une analyse de la sensibilité des résultats au seuil choisi permettrait de mieux identifier le seuil de développement urbain à partir duquel les changements sur la réponse hydrologique et/ou sur les paramètres du modèle sont significatifs. La richesse de la base de données développée peut permettre de mener une analyse plus approfondie sur le rôle de l'urbanisation en fonction de sa typologie. En effet, les caractéristiques urbaines entre la France et les États-Unis sont assez différentes (étalement plus important aux États-Unis mais concentration plus forte sur certains bassins français).

\subsection{Conclusion et perspectives}

Un modèle hydrologique horaire non dédié aux environnements urbanisés a été appliqué sur des bassins versants urbanisés afin d'estimer dans quelle mesure les paramètres de ce modèle sont sensibles aux aspects de l'urbanisation. Un échantillon de 365 bassins versants a été préparé, représentant 730 couples bassin-période de calage. L'analyse a été conduite sur une sélection de 416 bassin-période pour conserver l'homogénéité en termes de taille des bassins. Des indicateurs hydrologiques $\left(\frac{Q}{P}, B F I\right.$ et temps de réaction $\left.D T\right)$ ont été calculés sur ces périodes pour les mettre en relation avec les changements de valeurs des paramètres du modèle. Les résultats ont montré que les paramètres du modèle prennent des valeurs significativement différentes sur les bassins versants fortement ou faiblement urbanisés, surtout pour la capacité du réservoir de production $X_{1}$ et le temps de base des hydrogrammes unitaires $X_{4}$. Ceci est confirmé par les résultats sur le temps de réponse $D T$ et l'indice de l'écoulement de base $B F I$ ou encore sur le rendement $\frac{Q}{P}$.

Ce travail d'analyse de sensibilité "spatiale" des paramètres par rapport au degré d'urbanisation du bassin versant permet d'avoir une idée sur les changements à effectuer sur la structure du modèle. Une analyse "temporelle" de l'évolution dans le temps de chaque paramètre, bassin par bassin, compléterait les interprétations.

\section{Références}

Braud, I., Breil, P., Thollet, F., Lagouy, M., Branger, F., Jacqueminet, C., Kermadi, S., and Michel, K. (2013). Evidence of the impact of urbanization on the hydrological regime of a medium-sized periurban catchment in France. Journal of Hydrology, 485:5-23. 
Burns, D., Vitvar, T., McDonnell, J., Hassett, J., Duncan, J., and Kendall, C. (2005). Effects of suburban development on runoff generation in the Croton River basin, New York, USA. Journal of Hydrology, 311(1-4) :266-281.

Coron, L., Perrin, C., Delaigue, O., Thirel, G., Michel, C., Andréassian, V., Bourgin, F., Brigode, P., Le Moine, N., Mathevet, T., Mouelhi, S., Oudin, L., Pushpalatha, R., and Valéry, A. (2018). airGR : Suite of GR Hydrological Models for Precipitation-Runoff Modeling.

Diem, J. E., Hill, T. C., and Milligan, R. A. (2018). Diverse multi-decadal changes in streamflow within a rapidly urbanizing region. Journal of Hydrology, 556:61-71.

Edijatno, De Oliveira Nascimento, N., Yang, X., Makhlouf, Z., and Michel, C. (1999). GR3j : a daily watershed model with three free parameters. Hydrological Sciences Journal, 44(2):263-277.

Falcone, J. A. (2011). GAGES-II : Geospatial Attributes of Gages for Evaluating Streamflow. USGS Unnumbered Series, U.S. Geological Survey, Reston, VA.

Ficchi, A. (2017). An adaptive hydrological model for multiple time-steps : diagnostics and improvements based on fluxes consistency. PhD Thesis, Université Pierre et Marie Curie-Paris VI, Paris, France.

Furusho, C., Chancibault, K., and Andrieu, H. (2013). Adapting the coupled hydrological model ISBA-TOPMODEL to the long-term hydrological cycles of suburban rivers : Evaluation and sensitivity analysis. Journal of Hydrology, $485: 139-147$.

Gupta, H. V., Kling, H., Yilmaz, K. K., and Martinez, G. F. (2009). Decomposition of the mean squared error and NSE performance criteria : Implications for improving hydrological modelling. Journal of Hydrology, 377(1-2) :80-91.

Gupta, H. V., Perrin, C., Bloschl, G., Montanari, A., Kumar, R., Clark, M., and Andréassian, V. (2014). Large-sample hydrology : a need to balance depth with breadth. Hydrology and Earth System Sciences, 18(2) :p-463.

Gustard, A., Bullock, A., and Dixon, J. M. (1992). Low flow estimation in the United Kingdom. Number 108 in Report / Institute of Hydrology. Institute of Hydrology, Wallingford. OCLC : 730961823.

Hardegree, S. P., Van Vactor, S. S., Levinson, D. H., and Winstral, A. H. (2008). Evaluation of NEXRAD radar precipitation products for natural resource applications. Rangeland ecology \& management, 61(3) :346-353.

Hawley, R. J. and Bledsoe, B. P. (2011). How do flow peaks and durations change in suburbanizing semi-arid watersheds? A southern California case study. Journal of Hydrology, 405(1-2) :69-82.

Homer, C., Huang, C., Yang, L., Wylie, B., and Coan, M. (2004). Development of a 2001 national land-cover database for the United States. Photogrammetric Engineering \& Remote Sensing, 70(7) :829-840.

Horvat, D. J., Horvat, C. A., Calvert, C., and Crum, T. (2011). The Refreshed WSR-88 Level II Data Collection and Distribution Network. WSR-88D Radar Operations Center, Norman, Oklahoma.

Kjeldsen, T. R., Miller, J. D., and Packman, J. C. (2013). Modelling design flood hydrographs in catchments with mixed urban and rural land cover. Hydrology Research, 44(6) :1040-1057.

Le Moine, N. (2008). Le bassin versant de surface vu par le souterrain : une voie d'amélioration des performances et du réalisme des modèles pluie-débit? $\mathrm{PhD}$ Thesis, Université Pierre et Marie Curie-Paris VI, Paris, France. 
Le Moine, N., Andréassian, V., and Mathevet, T. (2008). Confronting surface- and groundwater balances on the La Rochefoucauld-Touvre karstic system (Charente, France). Water Resources Research, 44(3).

Livneh, B., Bohn, T. J., Pierce, D. W., Munoz-Arriola, F., Nijssen, B., Vose, R., Cayan, D. R., and Brekke, L. (2015). A spatially comprehensive, hydrometeorological data set for Mexico, the U.S., and Southern Canada 1950-2013. Scientific Data, 2 :150042.

Mathevet, T. (2005). Quels modèles pluie-débit globaux pour le pas de temps horaire? Développement empirique et comparaison de modèles sur un large échantillon de bassins versants. PhD Thesis, ENGREF (Paris), Paris, France.

Mejía, A., Rossel, F., Gironás, J., and Jovanovic, T. (2015). Anthropogenic controls from urban growth on flow regimes. Advances in Water Resources, 84 :125-135.

Oudin, L., Hervieu, F., Michel, C., Perrin, C., Andréassian, V., Anctil, F., and Loumagne, C. (2005). Which potential evapotranspiration input for a lumped rainfall-runoff model? Journal of Hydro$\log y, 303(1-4): 290-306$.

Oudin, L., Salavati, B., Furusho-Percot, C., Ribstein, P., and Saadi, M. (2018). Hydrological impacts of urbanization at the catchment scale. Journal of Hydrology, 559 :774-786.

$\mathrm{R}$ Core Team (2017). $\mathrm{R}$ : A language and environment for statistical computing.

Rose, S. and Peters, N. E. (2001). Effects of urbanization on streamflow in the Atlanta area (Georgia, USA) : a comparative hydrological approach. Hydrological Processes, 15(8):1441-1457.

Salavati, B., Oudin, L., Furusho, C., and Ribstein, P. (2015). Évaluation de l'impact de l'urbanisation sur la réponse hydrologique de 172 bassins versants américains. La Houille Blanche, (3):51-57.

Singh, R., Maheshwari, B., and Malano, H. M. (2009). Developing a conceptual model for water accounting in peri-urban catchments. In 18th World IMACS Congress and MODSIM09 International Congress on Modelling and Simulation. Modelling and Simulation Society of Australia and New Zealand and International Association for Mathematics and Computers in Simulation, Cairns, Australia, pages 13-17. 